\title{
A Real Time Road Sign Recognition using Neural Network
}

\author{
Mohammad Badrul Alam Miah \\ Dept. of Information and Communication Technology \\ Mawlana Bhashani Science and Technology University \\ Santosh, Tangail-1902, Bangladesh
}

\begin{abstract}
A current flow of interest is to recognize Road Signs. Road Signs are the most essential visual language of the world that represents some special circumstantial information of environment and provides significant information for guiding, warning people to make their movements safer, easier and more convenient. The proposed system introduces a real time Road sign recognition system with a new method to extract sign features. This system consists of three stages: image acquisition and preprocessing, feature extraction, and recognition. In the first stage, input image of Road sign are captured by digital camera with appropriate frame rate and then preprocessed image by using some image processing techniques, such as, gray scale conversion, noise reduction, normalization, median filtering, binarization, remove unwanted portion of image etc.. In second stage, a strong feature extraction method has been introduced to extract the some important feature of the input image. Finally, a multilayer neural network with back propagation learning algorithm is used to recognize the Road signs. The performance of the system is tested in different sorts of road signs and obtains the result where overall success rate of the system is $91.5 \%$ which meet the expectation the experimental of system.
\end{abstract}

\section{General Terms}

Computer Science, Neural Network.

\section{Keywords}

Computer Vision, Road Signs, Feature Extraction, Neural Network, Road Sign Recognition

\section{INTRODUCTION}

Driving of our country is a task based almost entirely on visual information processing. The road signs and traffic signals define a visual language interpreted by drivers. Road signs carry many information necessary for successful driving - they describe the current traffic situation, define right-of-way, prohibit or permit certain directions, warn about risky factors etc. This system focus on recognition of Road sign in real-time which is automatically recognizes road sign. Since that time, many research groups and companies are interested and conducted research in this field, and enormous amount of work has been done and knowledge of the field related to this research should be gathered. The proposed system consists of Image acquisition, Image Enhancement and Preprocessing, Feature Extraction, and Neural Network Recognition. The proposed system recognized the mandatory road signs like as following [1].

This paper is organized as follows: Section 2 explains Previous Works and Motivation of Road sign. The proposed system is described in section 3. In section 4 the Experimental Results and Performances are described. In section 5 Implementation is described. Finally the conclusion is outlined in Section 6.

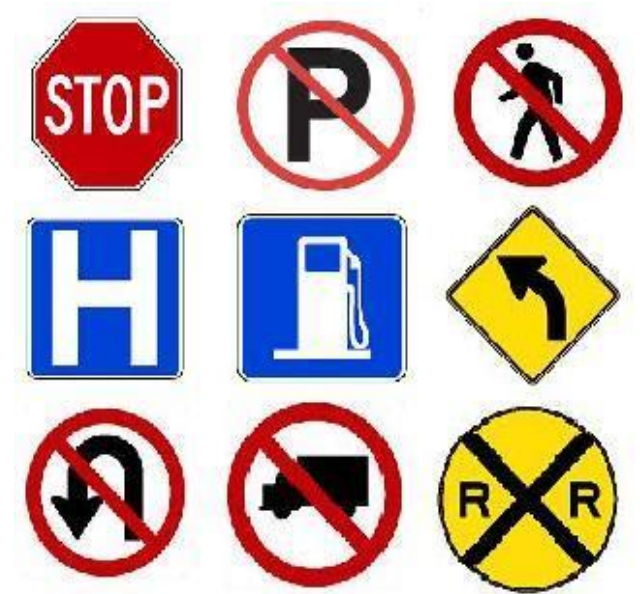

Fig 1: Mandatory Road Signs

\section{PREVIOUS WORKS and MOTIVATION}

Several researches have been made in the field of Road sign recognition system. Mueller et al. [2] developed a computer vision system for the detection of objects in outdoor scenes. Piccioli et al. [3] developed a road sign detection system by analyzing color as a priori information to limit the possible locations of signs in the image applying a cross-correlation technique. Novovicova et al. [4] used a Laplace Kernel classifier in the decision tree for road sign classification, despite some difficulties in the recognition of square signs in urban areas, where lots of horizontal and vertical lines exist in the image. Yuille et al. [5] developed an approach to detect only stop signs by correcting the color of the ambient illumination, locating the boundaries of the signs and mapping the sign into a front parallel position before reading the sign. De La Escalera et al. [6] started with color matching, in which they looked for patterns in specific relationships that correspond to triangular, rectangular or circular signs. Lauziere et al. [7] used a physics-based approach for sign recognition, but this approach required keeping in memory the changes in the model parameter to accommodate the natural variation of illumination. Lorsakul et al. [14] used Neural Network to recognize Traffic Sign for Intelligent Vehicle/Driver Assistance System. This system shows inconsistency results of traffic sign patterns with more complex background images. A. Ruta et al [12] developed an approach to detect Real-time traffic sign recognition from video by class specific discriminative features. Madhusudan Joshi et al [13] developed an approach to detect Automatic colored traffic sign detection using optoelectronic correlation architectures. Deshmukh et.al [11] Real-Time Traffic Sign Recognition System based on Color Image Segmentation. This system use segmentation technique that is more difficult tasks and the system has been implemented by using $\mathrm{C}$ language that is not 
so strong than MATLAB or other OOP. Saha et al [1] used Neural Network to recognize Road Sign. This system had implemented the task by using hybrid network (BAM and BPNN). This system uses more number of iteration that is depends on the number of hidden layer. The system took the hidden layer $70 \%$ of the input layer, the iteration was 88043 but it decreased to 43067 when we used $50 \%$ hidden layer. When the system used the hybrid network for the same percentage of hidden layer, it decreased from 34075 to 14977. After surveying different research works, the objective of proposed system is to represent a fast and robust system for road sign recognition which is real time. The proposed system use feature extraction methods to recognized road sign from Bangladeshi road sign properly.

\section{THE PROPOSED SYSTEM}

Many real-world computer vision applications require accurate detection of context-relevant objects from video images. Road sign recognition is a challenging example. Therefore and because of the high industrial relevance, many approaches for traffic sign detection and recognition have been proposed. The overall system architecture of the proposed system is shows in figure 2 . The whole system of road sign recognition divided into following steps-

- Image Acquisition.

- Image Preprocessing.

- Feature Extraction.

- Road Sign Recognition

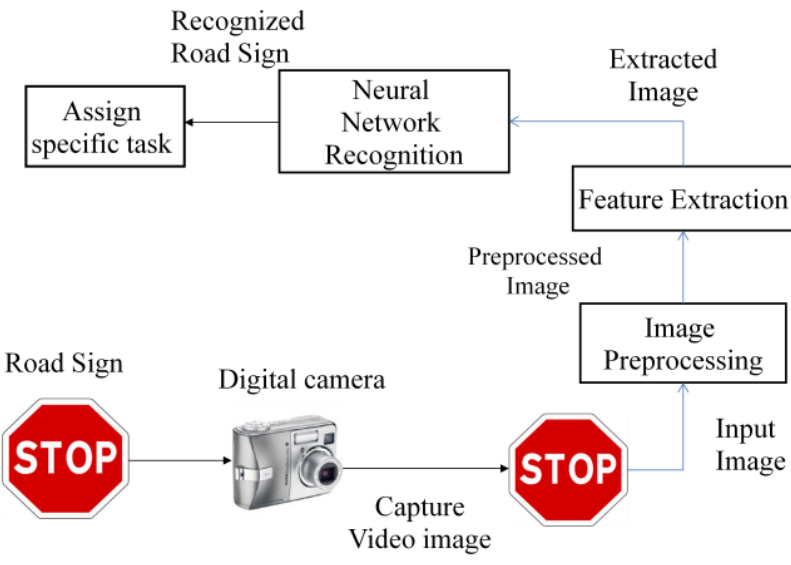

Fig 2: Road Sign Recognition System Architecture

\subsection{Image Acquisition}

A digital video camera is used to acquiesced Road sign Image. In this investigation, some mandatory road signs had been employed for image database in JPEG type file.

\subsection{Image Preprocessing}

Images that are acquiesce from video sequence, passed through the preprocessing steps. Figure 3 shows the block diagram of image preprocessing steps [15]. At first, RGB image converted into gray scale image using NTSC gray scale conversion which is frequently used to convert RGB to gray scale conversion shown in figure 4 . The equation is given bellow:

\section{Grayscale value $=0.3 \times$ Red $+0.59 \times$ Green $+0.11 \times$ Blue}

To remove the noise we use median filter [8]. The 'salt and pepper' noise which introduce during image acquisition is remove using this filter. Apply 3 × 3 square median filtering technique to remove noise shown in figure 5. Normalize the acquire image by converting it to $150 \times 140$ pixels. This size gives enough information of the image when the processing time is low. The image is converted from Gray scale to binary image that is an image with pixels 0's (white) and 1's (black) shown in figure 6 . This conversion can take place because it conveys proper information of Road Sign. Converting into binary image, we have to remove the unnecessary pixels (0) from original image. This is done because we need to develop size independent algorithm.

\section{Algorithm 1}

1) Start from top-left corner; repeat for each column and row.

If sum of all black pixels in row/column $>0$

Then save column and row

Else don't save column/row.

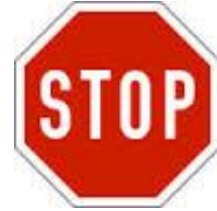

(a)

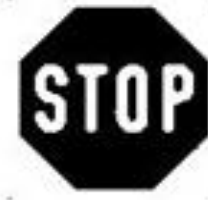

(b)
Fig 4: (a) RGB image (b) convert into gray scale image

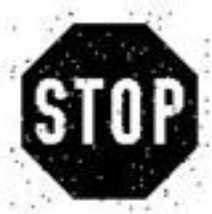

(a)

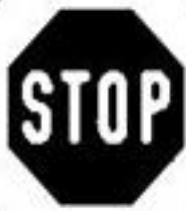

(b)
Fig 5: (a) Image with salt \& pepper noise, (b) remove noise

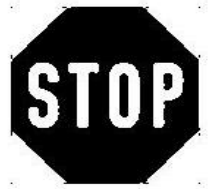

(a)

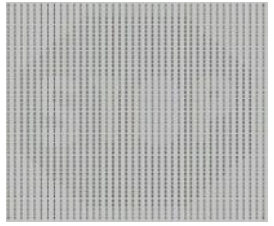

(b)
Fig 6: (a) Noise free gray scale image, (b) convert into Binary image

\subsection{Feature Extraction}

To extract the feature of the road sign I use a rotation and size independent feature extraction method and obtain 33 features for road sign.

\section{Center of the image}

Center of the image can obtain by using following equation:

Center_x $=$ width $/ 2 ; \quad$ Center_y $=$ height $/ 2$;

Feature1

The first feature is the relation between the height and the width of the road sign

feature1 $=$ height $/$ width

Feature 2 - 25

These features check how the black pixels are distributed in the image. First the number of pixels inside the image is 
calculated that is total_pixel of road signs. total_pixel $=$ height $\times$ weight.

The feature 2 and 3 are the percentage of black pixels located in the upper and lower areas of road signs.

feature2 $=$ up_pixels/total_pixels

feature $3=$ down_pixels $/$ total_pixels

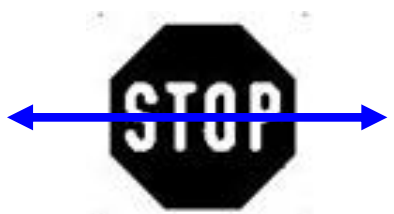

Fig 7: Divide the image into upper and lower area.

The feature 4 and 5 are the percentage of black pixels located in the left and right areas of road signs.

feature4 $=$ left_pixels $/$ total_pixels

feature $5=$ right_pixels/total_pixels

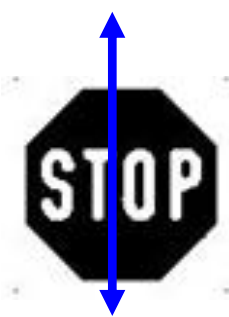

Figure 8: Divide images into left and right areas.

Similarly, now split the image into 4 sub regions and calculate the percentage of black pixels located in every region. Again sub divides every region into four and calculates the percentage of black pixels of those regions. The features 6 to 25 are the percentage of black pixels located in the every sub areas of road signs.

feature $_{n}=s u b \_a r e a \_p i x e l s_{n} /$ total_pixels; $\quad$ Where $\mathrm{n}=6$ to 25

\section{Feature 26}

The feature 26 is the average of the distance between all the black pixels and the central point.

$$
\text { feature } 26=\frac{1}{\text { Total_Pixels }} \times \sum_{y} \sum_{x} \sqrt{(x-i)^{2} \times(y-j)^{2}}
$$

Where $(\mathrm{i}, \mathrm{j})$ are the coordinates of a point and $(\mathrm{x}, \mathrm{y})$ are the coordinates of central point.

\section{Feature 27-33}

Calculate the central moments of road sign. For $f(x, y) 2$ dimntional function of $\mathrm{M} \times \mathrm{N}$ binary image, the moment of $\operatorname{order}(\mathrm{p}+\mathrm{q})$ is defined by: $m_{p q}=\sum_{x=1}^{M} \sum_{y=1}^{N}(x)^{p}(y)^{q} f(x, y)$

; Where $\mathrm{p}, \mathrm{q}=0,1,2,3 \ldots \ldots \ldots \ldots$

Central moment obtains by following equation:

$\mu_{p q}=\sum_{x} \sum_{y}(x-\bar{x})^{p}(y-\bar{y})^{q} f(x, y) ;$

Where $\bar{x}=\frac{m_{10}}{m_{00}}$ and $\bar{y}=\frac{m_{01}}{m_{00}}$
For scaling normalization the central moment changes as following equation:

$$
\eta_{p q}=\mu_{p q} / \mu_{00}^{\gamma} ; \quad \text { Where } \gamma=[(p+q) / 2]+1
$$

seven values, computed by normalizing central moments through order three, that are invariant to object scale, position, and orientation. In terms of the central moments, the seven moments are given as,

$$
\begin{aligned}
& M_{1}=\eta_{20}+\eta_{02} ; \quad M_{2}=\left(\eta_{20}-\eta_{02}\right)^{2}+4 \eta_{11}{ }^{2} ; \\
& M_{3}=\left(\eta_{30}-3 \eta_{12}\right)^{2}+\left(3 \eta_{21}-\eta_{03}\right)^{2} ; \\
& M_{4}=\left(\eta_{30}+\eta_{12}\right)^{2}+\left(\eta_{21}+\eta_{03}\right)^{2} \\
& M_{5}=\left(\eta_{30}-3 \eta_{12}\right)\left(\eta_{30}+\eta_{12}\right)\left(\left(\eta_{30}+\eta_{12}\right)^{2}-3\left(\eta_{21}+\eta_{03}\right)^{2}\right]+\left(3 \eta_{21}-\eta_{03}\right)\left(\eta_{21}+\eta_{03}\right)\left(3\left(\eta_{30}+\eta_{12}\right)^{2}-\left(\eta_{21}+\eta_{03}\right)^{2}\right] \\
& M_{6}=\left(\eta_{20}-\eta_{20}\right)\left[\left(\eta_{30}+\eta_{12}\right)^{2}-\left(\eta_{21}+\eta_{03}\right)^{2}\right]+4 \eta_{11}\left(\eta_{30}+\eta_{12}\right)\left(\eta_{21}+\eta_{03}\right) \\
& M_{7}=\left(3 \eta_{21}-\eta_{03}\right)\left(\eta_{30}+\eta_{12}\right)\left(\left(\eta_{30}+\eta_{12}\right)^{2}-3\left(\eta_{21}+\eta_{03}\right)^{2}\right]-\left(\eta_{30}+3 \eta_{12}\right)\left(\eta_{21}+\eta_{03}\right)\left(3\left(\eta_{30}+\eta_{12}\right)^{2}-\left(\eta_{21}+\eta_{03}\right)^{2}\right]
\end{aligned}
$$

\subsection{Road Sign Recognition}

Road sign recognition use neural network which is very efficient and reliable. Here explain network design, its parameters initialization, train and test images and implementation.

\subsubsection{Network Design}

A neural network is employed for road sign recognition. A multilayer feed forward neural network with supervised learning method is more reliable and efficient for this purpose. The network applies back propagation-learning algorithm that is a systematic method for training multiple layer ANNs. The objective of the training is to adjust the weight so that the input produce desired output.

\subsubsection{Train and Test Image}

Proposed system used four specific road sign images and five training samples for each type of road sign to train the network to recognize those signs properly. These images are static and taken from an image database.

Test images are collected by acquiring appropriate frame form video sequence, which capture instantly. Then these images are preprocessed for feature extraction to recognize which type of road sign it is.

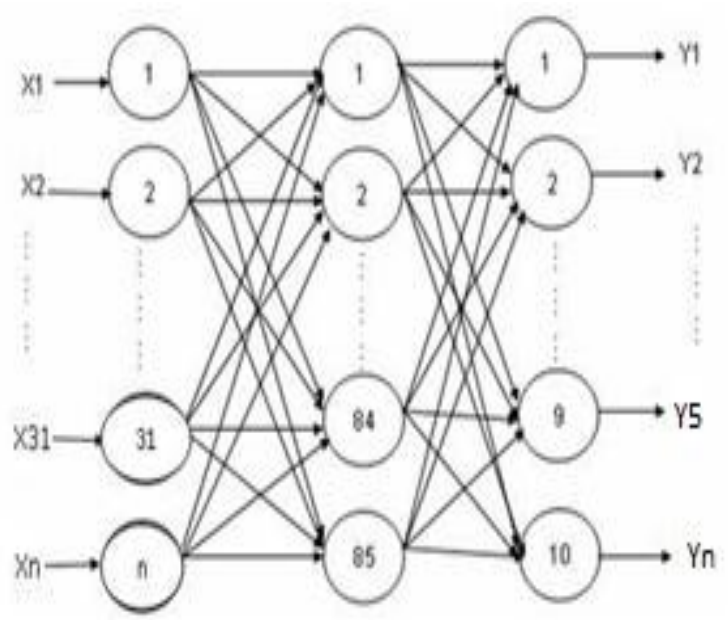

Fig 9: Network design for the system 


\section{EXPERIMENTAL RESULTS AND PERFORMANCES}

The experiment can take place by number of steps. At first input images are acquiring by sequence of video image. This image normalized into $150 \times 140$ pixels. Normalized image is converted into binary image to find the sign shape form the image. Now preprocessed image is passed through the feature extraction process. To extract the feature of the sign from an image get 33 elements for each input image. These input patterns are used to train the neural network and recognized Road signs. In this chapter will briefly explain the experiment and results that take perform on the Road sign recognition system.

\subsection{Train and Test Data}

To get efficient response from a network it is necessary to have proper number of training samples. To train the network use 5 samples for each type of Road sign. A single image contains 33 element vectors. A dataset of 20 images after feature extraction contains $33 \times 20$ elements. The figure 3.13 shows the training of the network. At the beginning the Mean squared error (MSE) is big, but after 127 epochs Neural network can recognize the training data with a very small error. To get the test data, used 40 frames form video sequence. To find the difference between two frames subtract one frame from its previous frame. If the value is grater then $30 \%$ then the frame is contain another type of Road sign. The achieve frames are then passed through the feature extraction process and get the test input patterns for recognition.

\subsection{Results}

The recognition method has been tested with 4 sign types. If the train and test set is same the recognition rates are above $99 \%$, otherwise the results are above $90 \%$. The overall recognition rates of test data are given bellow.

For each type of road sign, the system uses 10 frames and evaluates correct recognition rate and the error rate of the system. It has been used the following equation to find the correct recognition rate and the error rate:

Correct recognition $\%=$ correct_recognition / total_frames

\section{IMPLEMENTATION}

To develop a real-time road sign recognition system we use MATLAB, which is a high performance language for computing. MATLAB typically used in math and computation, algorithm development, modeling, simulation and prototyping, Data analysis, exploration, Scientific and engineering graphics and application development. It is the standard instructional tool for high-productivity research, development, and analysis. Toolboxes allow learning and applying specialized technology. Toolboxes are comprehensive collections of MATLAB functions that extend the MATLAB environment to solve particular classes of problems. It includes among others image processing and neural networks toolboxes. For these toolboxes, I decide, developing this thesis using MATLAB.

Table 1: Overall recognition rate of the system

\begin{tabular}{|c|c|c|c|}
\hline $\begin{array}{l}\text { Type of } \\
\text { Pattern }\end{array}$ & $\begin{array}{c}\text { No. of } \\
\text { frames }\end{array}$ & $\begin{array}{c}\text { Correct } \\
\text { recognition } \\
\text { rate }\end{array}$ & Error rate \\
\hline STOP & 10 & $95 \%$ & $5 \%$ \\
\hline & 10 & $90 \%$ & $10 \%$ \\
\hline & 10 & $94 \%$ & $6 \%$ \\
\hline
\end{tabular}

Error rate $=$ false_recognition $/$ total_frames

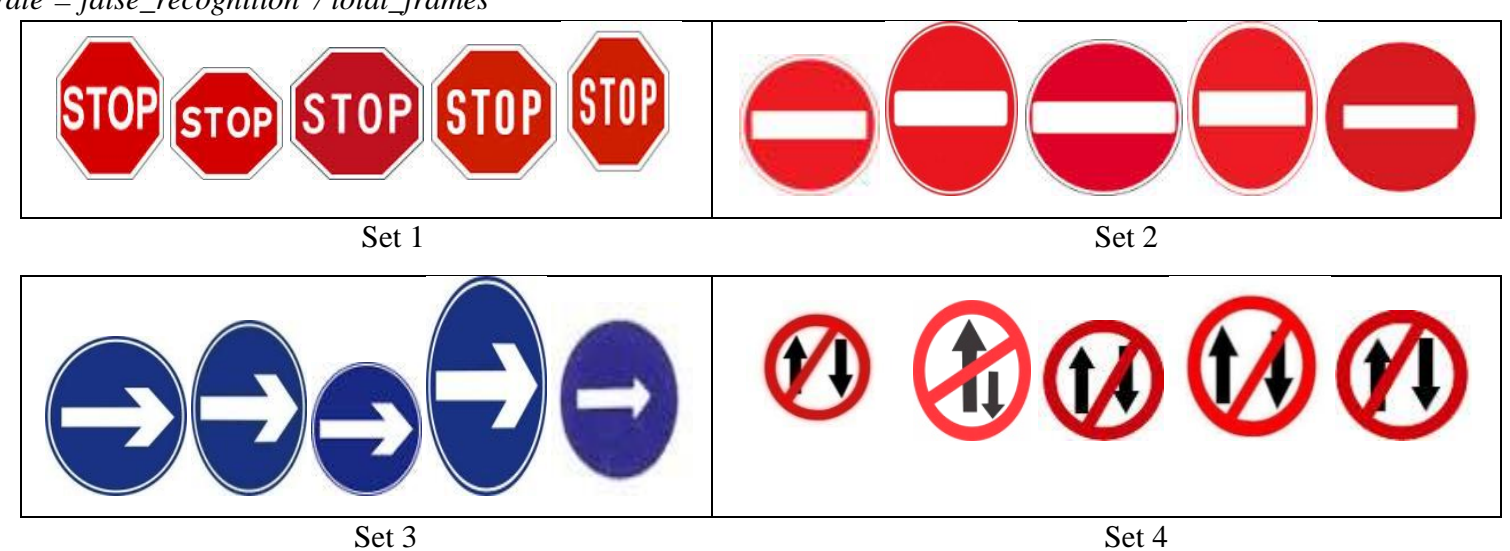

Fig 10: Train image for road sign 


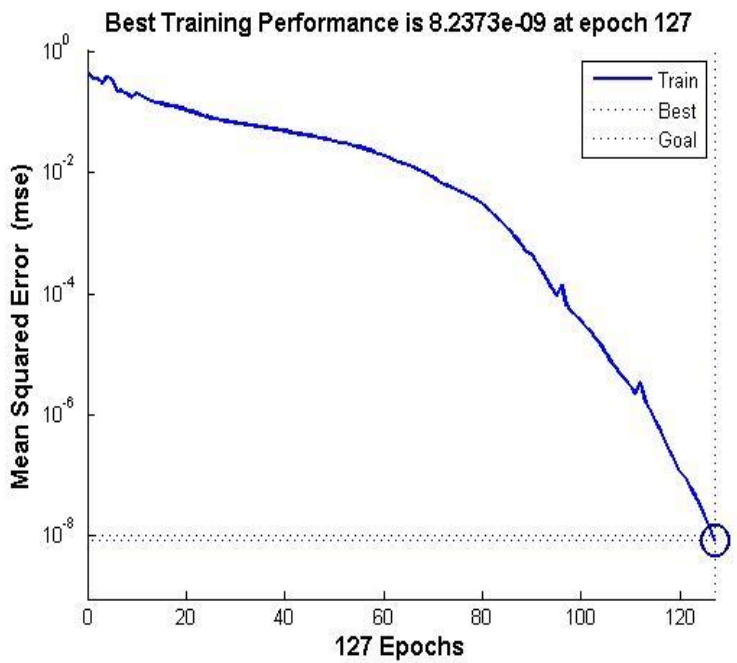

(a)

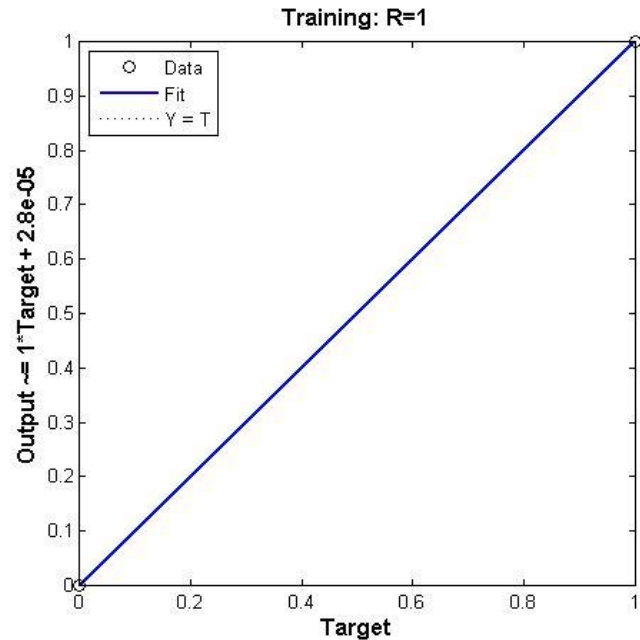

(b)

Fig 11: (a) performance Plot and (b) Regression Plot of the Neural Network

\section{CONCLUSION}

The system is tested 10 frames for each type of signs and obtains the result where overall success rate / result of the system is $91.5 \%$ which meet the expectation. At the end of this chapter, It is clearly say that the system achieve its desired expectation. The next chapter will describe the conclusion of the system.

\section{REFERENCES}

[1] Saha, S.K., Chakraborty, D., Al-Amin Bhuiyan, P.D.M., 2013, Neural Network based Road Sign Recognition, International Journal of Computer Applications (09758887) Volume 50 - No.10, July 2012 pp. 35-39.

[2] Mueller, R., Steck, M., 2003, -Road Sign Recognitionll, Term Paper, Computer Perception with Artificial Intelligence, University of Applied Sciences, Biel, Switzerland.

[3] Piccioli, G., De Micheli, E., Parodi, P., Campani, M., 1996. -Robust Method for Road Sign Detection and Recognitionll, Image and Vision Computing 14, pp.208223.

[4] Novovicova, J., Paclik, P., Pudil, P., and Somol, P., 2000. "Road Sign Classification Using Laplace Kernel Classifier," Pattern Recognition Letters 21, pp. 11651173.

[5] Yuille, A. L., Snow, D., and Nitzberg,M., 1998. -Using Color to Detect, Localize and Identify Informational Signsll, Proc. International Conference on Computer Vision ICCV98, Bombay, India, pp. 628-633.

[6] De la Escalera, A., Moreno, L., Salichs, M.A., and Amingol, J.M., 1997. "Road Traffic Sign Detection and Classification," IEEE Transactions Industrial Electronics, 44, pp. 848-859.

[7] Lauziere, Y., Gingras, D., Ferrie, F., 2001. -A Modelbased Road Sign Identification Systemll, Proc. IEEE
Computer Conference on Computer Vision and Pattern Recognition, pp. 1163-1170.

[8] Wikipedia, "Median filter," August 2006 , http://en.wikipedia.org/wiki/ Median_filter

[9] Mark S. Nixon, Alberto S. Aguado, 2002, Feature Extraction and Image Processing, First edition, Great Britain: British Library Cataloguing in Publication Data, pp. 249, 161-164

[10] Rafael C. Gonzalez and Richard E. Woods, 2003, Digital Image Processing and Analysis, Second Edition, India: Pearson Education Asia, pp.233-235, 341

[11] Deshmukh, V. R., Patnaik, G. K., Patil, M. E.,-2013, "Real-Time TrafficSign Recognition System based on Colour Image Segmentation", International Journal of Computer Applications (0975 - 8887) Volume 83 No3, December 2013, pp.30-34.

[12] A. Ruta and X. YongminLi, 2010, "Real-time traffic sign recognition from video by class specific discriminative features," Pattern Recognition, pp. 416430.

[13] Madhusudan Joshi, Mohan Jeet Singh, and Saurabha Dalela, 2008, Automatic colored traffic sign detection using optoelectronic correlation architectures, IEEE conference on Vehicular Electronics and Safety USA, Sept 22-24, 2008.

[14] Lorsakul, A., Suthakorn, J., 2007, Traffic Sign Recognition for Intelligent Vehicle/Driver Assistance System Using Neural Network on OpenCV, The 4th International Conference on Ubiquitous Robots and Ambient Intelligence (URAI 2007).

[15] Alam Miah, M.B., Anamul Haque, S.M., Mazumder, R., Rahman, M.Z., -Nov 2011, "A New Approach for Recognition of Holistic Bangla Word using Neural Network", International Journal of Data Warehousing \& Mining, Vol-1, issue-2, pp. 135-141. 